\title{
Validating the performance of the Empirical Canadian High Arctic Ionospheric Model (E-CHAIM) with in situ observations from DMSP and CHAMP
}

\author{
David R. Themens*, P. Thayyil Jayachandran, and Anthony M. McCaffrey \\ Department of Physics, University of New Brunswick, Fredericton, NB, Canada
}

Received 26 November 2018 / Accepted 22 May 2019

\begin{abstract}
The Empirical Canadian High Arctic Ionospheric Model (E-CHAIM) is a new empirical model of high latitude ionospheric electron density. While the introductory studies regarding E-CHAIM include validations, E-CHAIM's topside model was notably excluded from independent validation using datasets not included in the model fitting. In this study, we undertake such a validation using in situ electron density observations from the Defense Meteorological Satellite Program (DMSP) constellation of satellites and the Challenging Mini-satellite Payload (CHAMP) mission. Through this validation, we show that E-CHAIM generally outperforms the International Reference Ionosphere (IRI) at DMSP orbit ( $\sim 830 \mathrm{~km}$ altitude), with RMS errors of 8.3-9.8 $\times 10^{9} \mathrm{e} / \mathrm{m}^{3}$ versus the IRI's $1.2-1.3 \times 10^{10} \mathrm{e} / \mathrm{m}^{3}$. E-CHAIM's improvement over the IRI is consistent at all latitudes but is particularly noted in sub-auroral regions and is mainly limited to summer and equinox periods. At CHAMP orbit, E-CHAIM and the IRI are found to perform largely comparably, with E-CHAIM outperforming the IRI only marginally with RMS errors of $7.11 \times 10^{10} \mathrm{e} / \mathrm{m}^{3}$ versus the IRI's $7.48 \times 10^{10} \mathrm{e} / \mathrm{m}^{3}$. This improvement is found to be largely constrained to sub-auroral latitudes with both models performing comparably at higher latitudes. An observed tendency for the IRI to overestimate electron density in the near-peak (at CHAMP orbit) and underestimate electron density at higher altitudes (DMSP orbit) appears to be consistent with previous work, which identified this pattern to result from shortcomings in the NeQuick topside function curvature at high latitudes.
\end{abstract}

Keywords: Ionosphere (auroral) / Ionosphere (polar) / Validation / Radio Sciences / Ionosphere (mid latitude)

\section{Introduction}

The Empirical Canadian High Arctic Ionospheric Model (E-CHAIM) is a relatively new empirical representation of high latitude $\left(>50^{\circ} \mathrm{N}\right.$ geomagnetic latitude) electron density (Themens et al., 2017a, 2018). The model was built as a stand-alone replacement for the use of the International Reference Ionosphere (IRI) in these regions and features significant improvements over the IRI in terms of $h_{\mathrm{m}} F 2$ and $N_{\mathrm{m}} F 2$ (Themens et al., 2017a). Unfortunately, due to a limited dataset and the desire to use all available topside electron density profiles for the fitting of the model, independent validation of the E-CHAIM topside has not yet been undertaken. It is the intent of this study to provide this independent validation.

Accurate topside electron density modeling is integral to several ionospheric model applications, serving as the dominant contribution to ionospheric total electron content (TEC),

*Corresponding author: david. themens@gmail.com particularly at high latitudes (Themens et al., 2014, Themens \& Jayachandran, 2016; Bjoland et al., 2016). Themens \& Jayachandran (2016) demonstrated that IRI suffers significant errors in modeling this electron density at high latitudes, sometimes underestimating the integrated topside electron density by up to six TECU $\left(1\right.$ TECU $\left.=10^{16} \mathrm{e} / \mathrm{m}^{3}\right)$. In that study, the authors demonstrated that, while foF2 errors contributed somewhat to these errors, up to four TECU of the remaining error stemmed from the topside shape function. These results combined with further diagnostics completed in Themens et al. (2017b) lead the designers of E-CHAIM develop a new topside shape parameterization, built on the successes of the IRI's NeQuick topside function (Coïsson et al., 2006) but modified to provide an improvement in topside curvature and thickness. In this parameterization, topside electron density is represented by the following function:

$$
N(h)=\frac{4 N_{\mathrm{m}} F 2}{(1+\exp (z))^{2}} \exp (z),
$$




$$
\begin{gathered}
z=\frac{h-h_{\mathrm{m}} F 2}{H}, \\
H=H_{0}\left[1+\frac{r g\left(h-h_{\mathrm{m}} F 2\right)}{r H_{0}+g\left(h-h_{\mathrm{m}} F 2\right)}\right],
\end{gathered}
$$

where $N_{\mathrm{m}} F 2$ is the peak density of the layer, $h$ is altitude, $h_{\mathrm{m}} F 2$ is the height of the layer peak, $\mathrm{H}_{\mathrm{o}}$ is the scale height at the F2-peak, $r$ is a parameter controlling the asymptote of the scale height at high altitudes, and $g$ is a parameter controlling the rate of increase in scale height near the F2 peak (Coïsson et al., 2006; Nava et al., 2008). In the E-CHAIM implementation of this model, the $r$ and $g$ parameters were modified to $(20,0.18)$ in place of the NeQuick's $(100,0.125)$ and an independent spherical cap harmonic model was developed to represent $H_{0}$ (Themens et al., 2018).

While the above changes were demonstrated to provide a significant improvement with respect to the fitting dataset, further validation against independent datasets is necessary. For this purpose, we have here gathered over a decade of in situ electron density observations from the Defense Meteorological Satellite Program (DMSP) constellation of satellites and the Challenging Mini-satellite Payload (CHAMP) mission. These instruments have been used previously to evaluate the performance of other empirical models, like the IRI and NeQuick, on global scales. Lühr \& Xiong (2010) used CHAMP and Gravity Recovery and Climate Experiment (GRACE) in situ electron density measurements to demonstrate that the IRI overestimates globally averaged electron density in near-peak topside. Xiong et al. (2013) similarly used CHAMP and GRACE data to demonstrate that the IRI overestimates topside electron density in the Main Ionospheric Trough (MIT) region. Migoya-Orue et al. (2013) used in situ measurements from the DMSP F13 and F15 satellites to demonstrate that the IRI's NeQuick option tends to underestimate electron density at DMSP orbit at high latitudes and that the Corrected-IRI2001 option tends to overestimate the density in this region. Here we will focus on the validation of E-CHAIM but will also compare to the IRI's performance for context.

In Section 2, we provide an overview of the data used in this study and the two models of interest, namely the IRI and E-CHAIM. In Section 3 we present a comparison between DMSP in situ measurements and coincident IRI/E-CHAIMmodeled electron density, with a corresponding comparison to CHAMP measurements in Section 4. We finish in Sections 5 and 6 with a discussion of the results and some conclusions, respectively.

\section{Data}

\subsection{DMSP in situ electron density}

DMSP satellites orbit in a Sun-synchronous, circular orbit at between $830 \mathrm{~km}$ and $880 \mathrm{~km}$ with an orbital period of $\sim 110 \mathrm{~min}$ (Garner et al., 2010). For this study, due to the redundancy of the orbit of some satellites, only the F17 and F18 DMSP satellites are used. This data was gathered from the https://satdat. ngdc.noaa.gov/dmsp/data/ data portal.

DMSP has featured the Special Sensor for Ions, Electrons, and Scintillation (SSIES) instrument package on satellite payloads since the late 1980s. The instruments within this package are jointly capable of determining several plasma properties, the most pertinent to this study being total plasma density $(\mathrm{Ne})$. The main instruments used in this study are the Retarding Potential Analyzer (RPA) and Faraday Scintillation Cup instruments, which are both capable of producing accurate in situ total plasma density (electron density) measurements.

The RPA and scintillation cup instruments are capable of producing a measurement of electron density at $0.25 \mathrm{~Hz}$ and $24 \mathrm{~Hz}$ sampling, respectively. As this study is only interested in relatively low-resolution variabilities (both in time and space) and to reduce the overwhelming size of the dataset, only electron density measurements every $20 \mathrm{~s}$ are used. This sampling resolution translated spatially into a latitude resolution of $\sim 1^{\circ}$ and an MLT resolution $\leq 15 \mathrm{~min}$, where MLT resolutions are greatest at lower latitudes due to the orbit conformation.

To ensure the quality of the dataset, we have undertaken the same quality control measures used in Garner et al. (2010). This includes their threshold checks of $2 \times 10^{8}<\mathrm{Ne}<10^{12}$ for both the scintillation cup and RPA instruments and their self consistency checks between RPA and scintillation cup measurements for ranging error catching. See Garner et al. (2010) for further details. Unfortunately, due to having used the SSIES Environmental Data Record files rather than the binary data, it was not possible to undertake the RPA quality control measures that depended on having the raw RPA data.

A plot of the magnetic local time and geomagnetic latitude distribution of the F17 and F18 datasets is presented in Figure 1. Note the $\sim 2$-h difference in the locations of the distribution maxima in MLT between F17 and F18, with F18 orbiting predominantly 2-h MLT later than F17.

\subsection{CHAMP in situ electron density}

CHAMP, an effort of the German Research Centre for Geosciences, Potsdam, (GFZ) and the German Aerospace Centre (DLR), was launched into a near-circular $456 \mathrm{~km}$ polar orbit (inclination $87.3^{\circ}$ ) on July 15th, 2000 and re-entered the Earth's atmosphere in September 2010. The satellite orbit is such that the local time of the satellite changes by $1 \mathrm{~h}$ every $\sim 11$ days, covering all local time sectors after $\sim 130$ days of orbit. The CHAMP data was gathered from the GFZ data portal at http://isdc.gfz-potsdam.de/champ/CH-ME-2-PLP.

Of primary interest to ionospheric researchers, CHAMP featured a magnetometer for measuring the Earth's main field, several GPS radio occultation receivers for inverting profiles of ionospheric electron density, and a Planar Langmuir Probe (PLP) to measure in situ electron density. This PLP instrument was used to determine in situ electron density along the CHAMP orbit at $15 \mathrm{~s}$ resolution. In this study, only data processed using the latest method (processing ID 21) and which was recorded above $50^{\circ} \mathrm{N}$ geomagnetic latitude is used. A contour map of the data distribution used in this study is presented in Figure 2.

\subsection{E-CHAIM}

The topside and F2-peak components of the E-CHAIM model were first presented in Themens et al. (2017a, 2018). The model is a regional solution for locations above $50^{\circ} \mathrm{N}$ geomagnetic latitude and was designed as an alternative to the 


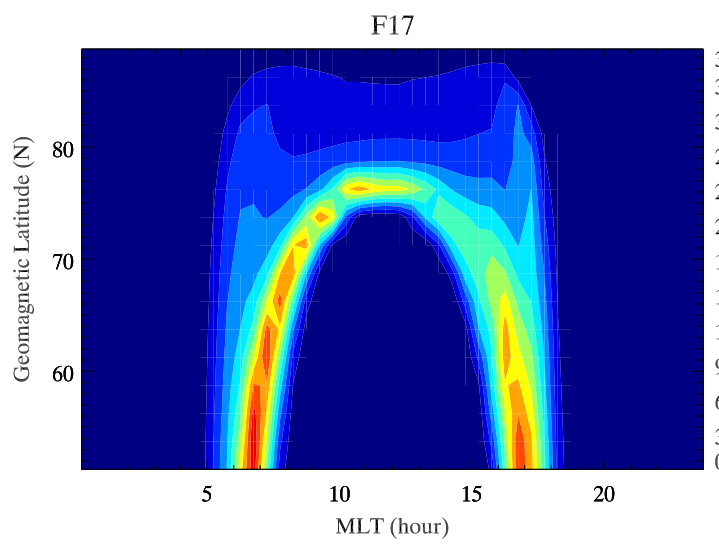

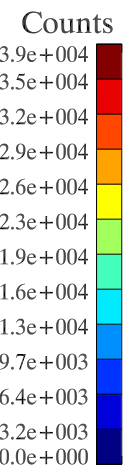

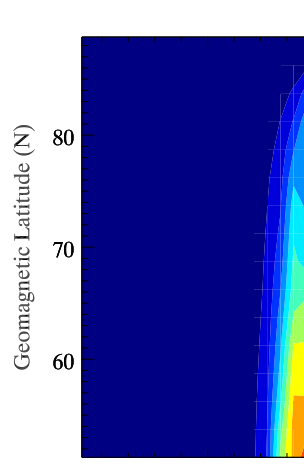

F18

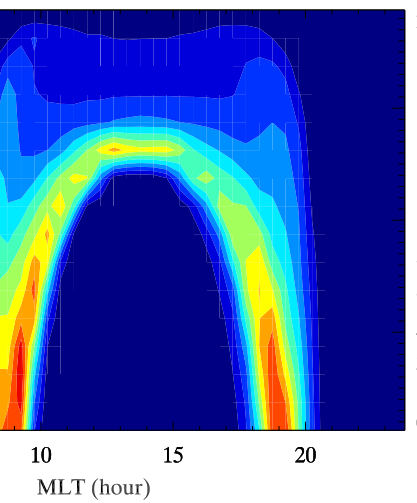

Counts

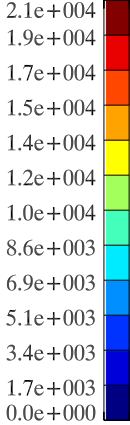

$0.0 \mathrm{e}+000$

MLT (hour)

Fig. 1. Distribution of DMSP F17 (left) and F18 (right) data with respect to Geomagnetic Latitude and Magnetic Local Time (MLT). For the purpose of these statistics, bins sizes were $0.25 \mathrm{~h}$ in MLT and $2.5^{\circ}$ in geomagnetic latitude.

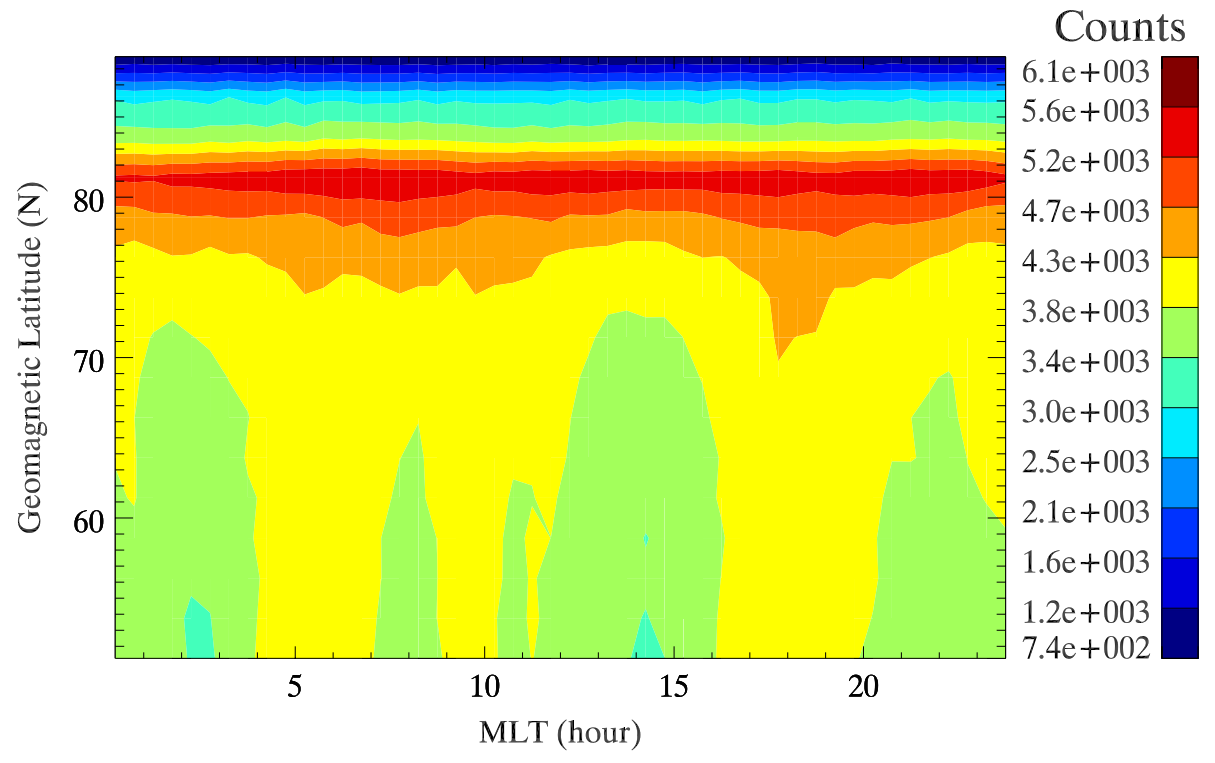

Fig. 2. Distribution of CHAMP data with respect to Geomagnetic Latitude and Magnetic Local Time (MLT). For the purpose of these statistics, bins sizes were $0.25 \mathrm{~h}$ in MLT and $2.5^{\circ}$ in geomagnetic latitude.

use of the IRI at high latitudes. The model is free and openly available online at https://e-chaim.chain-project.net. The E-CHAIM distribution features source code versions provided in Matlab, IDL, and C, as well as a website interface, similar to that available for the IRI. The most recent version of E-CHAIM (v1.2.1) is used for this study.

E-CHAIM was developed using an extensive dataset of ionosondes, topside sounders, Incoherent Scatter Radars (ISRs), and Radio Occultation (RO) satellites. The topside in E-CHAIM is represented by a modified version of the NeQuick topside function anchored at the F2-region peak density $\left(N_{\mathrm{m}} F 2\right)$ and height $\left(h_{\mathrm{m}} F 2\right)$ (Themens et al., 2018). The model is fit to basis sets of spherical cap harmonics for spatial variability and Fourier expansions in day of year for seasonal variability. For diurnal variations, the $h_{\mathrm{m}} F 2$ and $N_{\mathrm{m}} F 2$ models were fit independently for each UTC hour, while the topside thickness model uses local time as its longitude coordinate and functions of solar zenith angle to accommodate its diurnal variability. Both the $N_{\mathrm{m}} F 2$ and topside thickness models also feature accommodations for the effect of ionospheric storms.

\subsection{IRI}

The IRI is the internationally recognized standard for ionospheric specification (Bilitza et al., 2011), built through a collaboration between International Union of Radio Science (URSI) and the Committee on Space Research (COSPAR) over several decades (Bilitza, 1990, 2001; Bilitza et al., 2000, 2011, 2012; Bilitza \& Reinisch, 2008). The model features several options for $N_{\mathrm{m}} F 2, h_{\mathrm{m}} F 2$, and topside shape. For the purpose of this study, the Shubin (2015) option was selected for $h_{\mathrm{m}} F 2$, as the default AMTB-2013 model was not intended for use above $60^{\circ} \mathrm{N}$ geomagnetic latitude (Altadill et al., 2013), and the URSI option was selected for foF2, as it is generally 

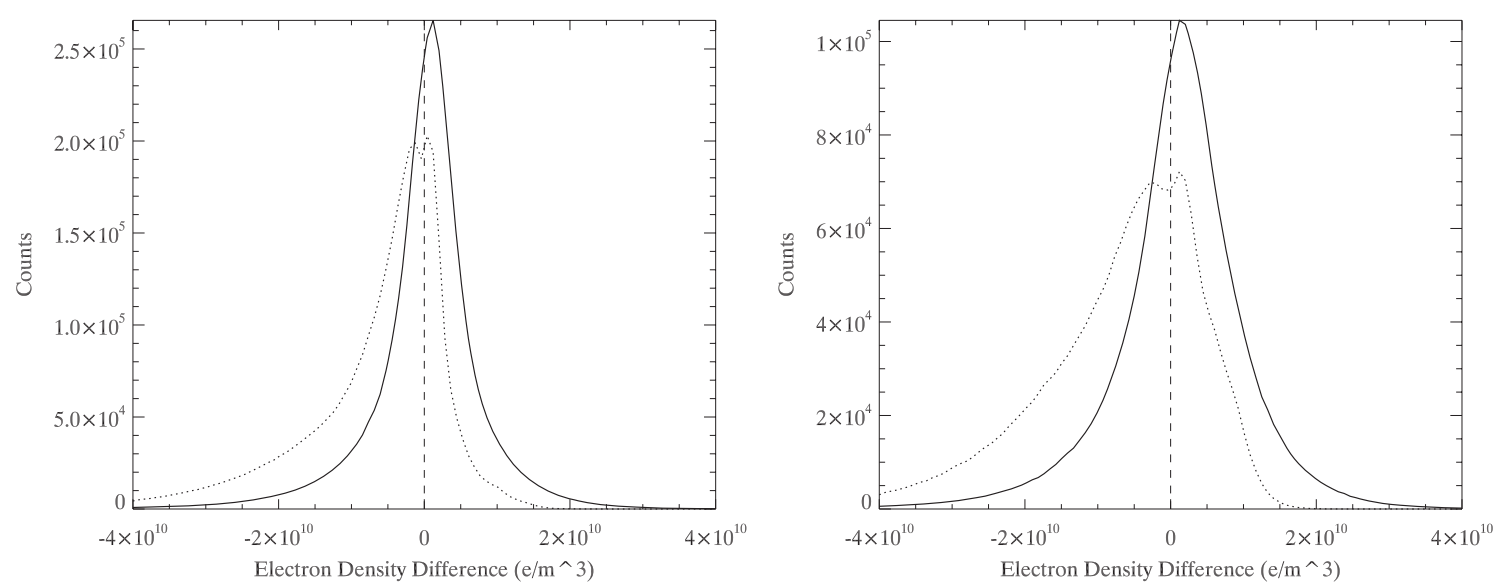

Fig. 3. Probability distributions of electron density errors for the IRI (dotted lines) and E-CHAIM (solid lines) with respect to DMSP in situ electron density data for satellite F17 (left) and satellite F18 (right).

found to be the better performing IRI foF2 option at high latitudes (Themens et al., 2014). The NeQuick option, the IRI's default, was selected for the topside representation (Nava et al., 2008).

Based on the results of Themens et al. (2014, 2018), which demonstrate an underestimation of $N_{\mathrm{m}} F 2$ by the IRI and a tendency to overestimate the near-peak topside thickness while underestimating electron density aloft, we expect the IRI's $N_{\mathrm{m}} F 2$ and topside thickness errors to negate each other to some extent in the near-peak region but result in an additive underestimation of electron density at high altitudes (beginning above approximately $\left.h_{\mathrm{m}} F 2+200 \mathrm{~km}\right)$.

\section{Validating E-CHAIM with DMSP}

\subsection{Overall comparison}

The process of validating E-CHAIM with DMSP in situ data begins by first examining the overall distribution of error performance over the entire dataset. This type of comparison can be misleading due to the anisotropic sampling in local time that occurs at the polar portion of the DMSP orbit, but nonetheless these average statistics are presented to begin the discussion. For F17, the time range used is January 2007February 2017, and for F18, the time range used is January 2012-February 2017.

From Figure 3, first note largely symmetric error statistics from E-CHAIM with the peak of the error distribution indicating a slight overestimation trend. For the IRI, highly skewed error distribution toward underestimation with a double peak feature near the distribution maximum is observed. This double peak is likely the result of two separate error populations, the nature of which will be discussed as portions of the dataset are isolated for more informative comparisons. One may consult Migoya-Orue et al. (2013) for further discussion regarding the statistical behaviour of the IRI with respect to DMSP observations, as our main focus here is to evaluate the performance of E-CHAIM. Regardless, from Table 1 we see that E-CHAIM appears to outperform the IRI on the average when examining RMS errors over the entire dataset.
Table 1. E-CHAIM and IRI Mean and RMS errors for DMSP satellites F17 and F18.

\begin{tabular}{lcc}
\hline & Mean error $\left(\mathrm{e} / \mathrm{m}^{3}\right)$ & RMS $\left(\mathrm{e} / \mathrm{m}^{3}\right)$ \\
\hline IRI F17 & $-6.0 \mathrm{E}+09$ & $1.2 \mathrm{E}+10$ \\
E-CHAIM F17 & $2.9 \mathrm{E}+08$ & $8.3 \mathrm{E}+09$ \\
IRI F18 & $-6.0 \mathrm{E}+09$ & $1.3 \mathrm{E}+10$ \\
E-CHAIM F18 & $8.0 \mathrm{E}+08$ & $9.8 \mathrm{E}+09$ \\
\hline
\end{tabular}

\subsection{Morning and evening seasonal and solar cycle variations}

To better identify the strengths and shortcomings of each model, the performance of each model is examined in their representation of monthly averages in a series of isolated domains. Because of the orbital conformation of the DMSP satellites, note from Figure 1 that the dataset is generally isolated to MLT bands for lower latitudes, but the transit across the pole leads to a wide variation in MLT at higher latitudes. For the following assessment, the narrow bands in MLT in two latitude bins $\left(50^{\circ}-60^{\circ} \mathrm{N}\right.$, and $60^{\circ}-70^{\circ} \mathrm{N}$ geomagnetic latitude) are examined before later proceeding to examine monthly median behaviour in a $70^{\circ}-80^{\circ} \mathrm{N}$ geomagnetic latitude bin. For satellite F17, the bands of MLT used are 5.5-7.5 and 16-18 h MLT. For F18, we examine MLT hours 7.5-9.5 and 18-20. These bands have been chosen to center on the polar transition of the DMSP orbit, as well as to center on regions best sampled by the DMSP orbit. In Figure 4, the monthly average electron density at DMSP orbit for these MLT and geomagnetic latitude bins are presented for satellite F17.

From this figure, one can clearly see that the main improvement of E-CHAIM over the IRI is due to E-CHAIM's increased capacity to represent the seasonal variation in electron density along the DMSP orbit. E-CHAIM does an excellent job matching the monthly average electron density trend of the DMSP data, with particular improvement during summer periods and at high solar activity. The IRI fails to capture the amplitude of the seasonal and solar cycle trend in the DMSP observations, generally underestimating electron density during summer and 
50-60 MLAT, 5.5-7.5 MLT

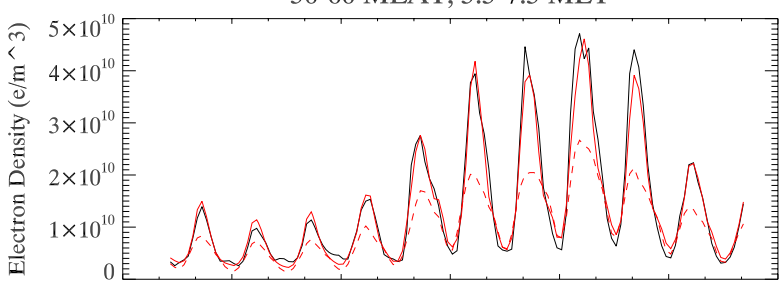

Jan, 2006 Jan, 2008 Jan, 2010 Jan, 2012 Jan, 2014 Jan, 2016 Jan, 2018

50-60 MLAT, 16-18 MLT

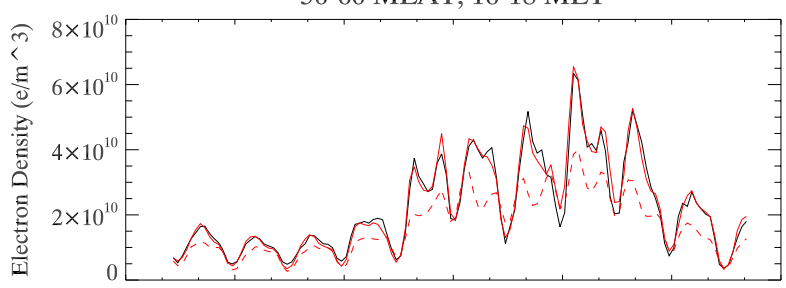

Jan, 2006 Jan, 2008 Jan, 2010 Jan, 2012 Jan, 2014 Jan, 2016 Jan, 2018

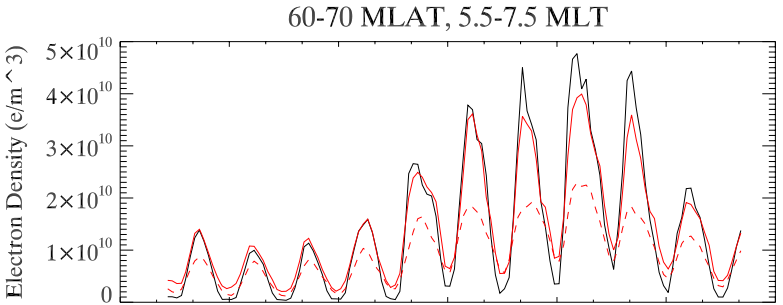

Jan, 2006 Jan, 2008 Jan, 2010 Jan, 2012 Jan, 2014 Jan, 2016 Jan, 2018

60-70 MLAT, 16-18 MLT

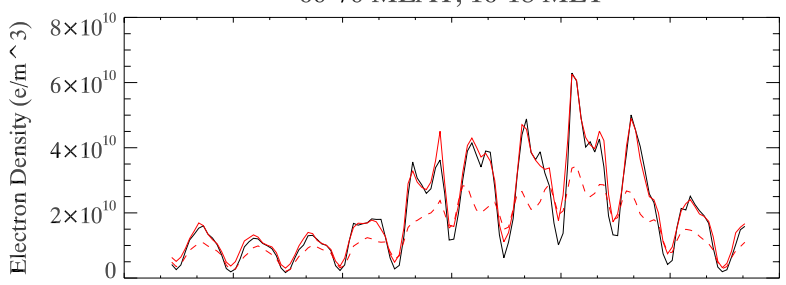

Jan, 2006 Jan, 2008 Jan, 2010 Jan, 2012 Jan, 2014 Jan, 2016 Jan, 2018

Fig. 4. Plots of monthly mean electron density from DMSP F17 (black line) and the E-CHAIM (red solid line) and IRI (red dashed line) models at the DMSP F17 satellite orbit. The top plots correspond to the 5.5-7.5 MLT bin, the bottom plots correspond to the 16-18 MLT bin, the left plots are for the $50-60^{\circ} \mathrm{N}$ geomagnetic latitude bin, and the right plots are for the $60-70^{\circ} \mathrm{N}$ geomagnetic latitude bin.

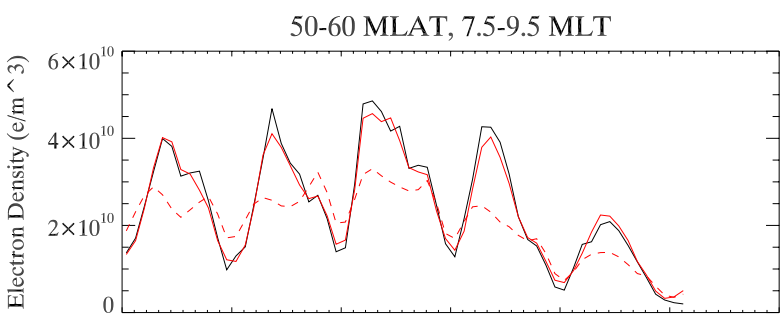

Jan, 2012 Jan, 2013 Jan, 2014 Jan, 2015 Jan, 2016 Jan, 2017 Jan, 2018 50-60 MLAT, 18-20 MLT

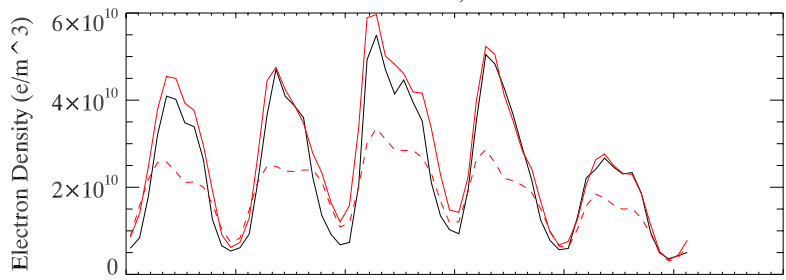

Jan, 2012 Jan, 2013 Jan, 2014 Jan, 2015 Jan, 2016 Jan, 2017 Jan, 2018

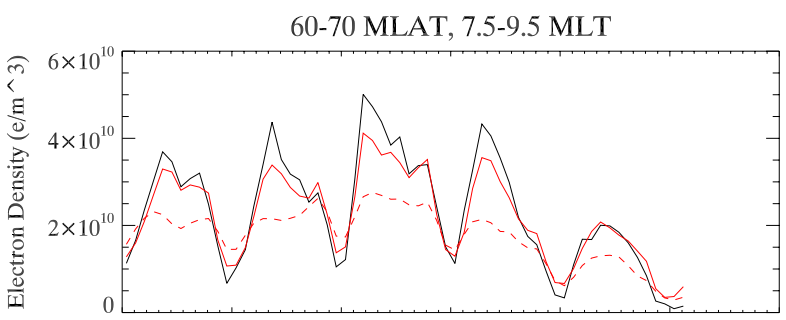

Jan, 2012 Jan, 2013 Jan, 2014 Jan, 2015 Jan, 2016 Jan, 2017 Jan, 2018 60-70 MLAT, 18-20 MLT

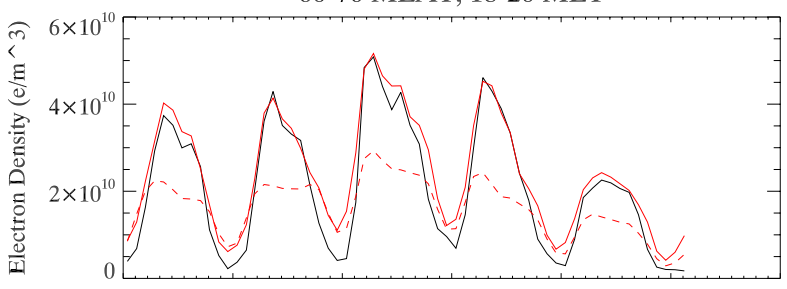

Jan, 2012 Jan, 2013 Jan, 2014 Jan, 2015 Jan, 2016 Jan, 2017 Jan, 2018

Fig. 5. Same as Figure 4, but for DMSP satellite F18. The top plots correspond to the 7.5-9.5 MLT bin, the bottom plots correspond to the 18-20 MLT bin, the left plots are for the $50^{\circ}-60^{\circ} \mathrm{N}$ geomagnetic latitude bin, and the right plots are for the $60^{\circ}-70^{\circ} \mathrm{N}$ geomagnetic latitude bin.

equinox periods, particularly at high solar activity. For the morning MLT bins (5.5-7.5 MLT), note a slight tendency for E-CHAIM to underestimate electron density during summer periods at high solar activity, particularly in the higher latitude bin $\left(60^{\circ}-70^{\circ} \mathrm{N}\right.$ geomagnetic latitude), and converge to the IRI trend of overestimating the electron density during winter periods in the higher latitude bin. For the evening MLT bins (16-18 MLT), note almost perfect performance by E-CHAIM at both latitudes during summer and equinox periods with again a slight tendency to overestimate electron density during the winter, converging to the IRI curve. The IRI appears to again underestimate summer and equinox electron density but also somewhat exaggerates the semi-annual anomaly at high solar activity. The IRI's tendency to perform well during winter periods while significantly underestimating electron density during equinox and summer periods is the main culprit behind the double peak in the IRI's error distribution in Figure 3.

The same monthly averages of Figure 4, but for satellite F18, are presented in Figure 5.

As you may note, both E-CHAIM and the IRI again provide similar electron densities during winter periods, culminating in a slight overestimation with respect to DMSP data, but E-CHAIM again significantly outperforms the IRI during summer and equinox periods in all MLT and geomagnetic latitude bins. Overall, the results for satellite F18 are very similar, qualitatively, to those from satellite F17. 

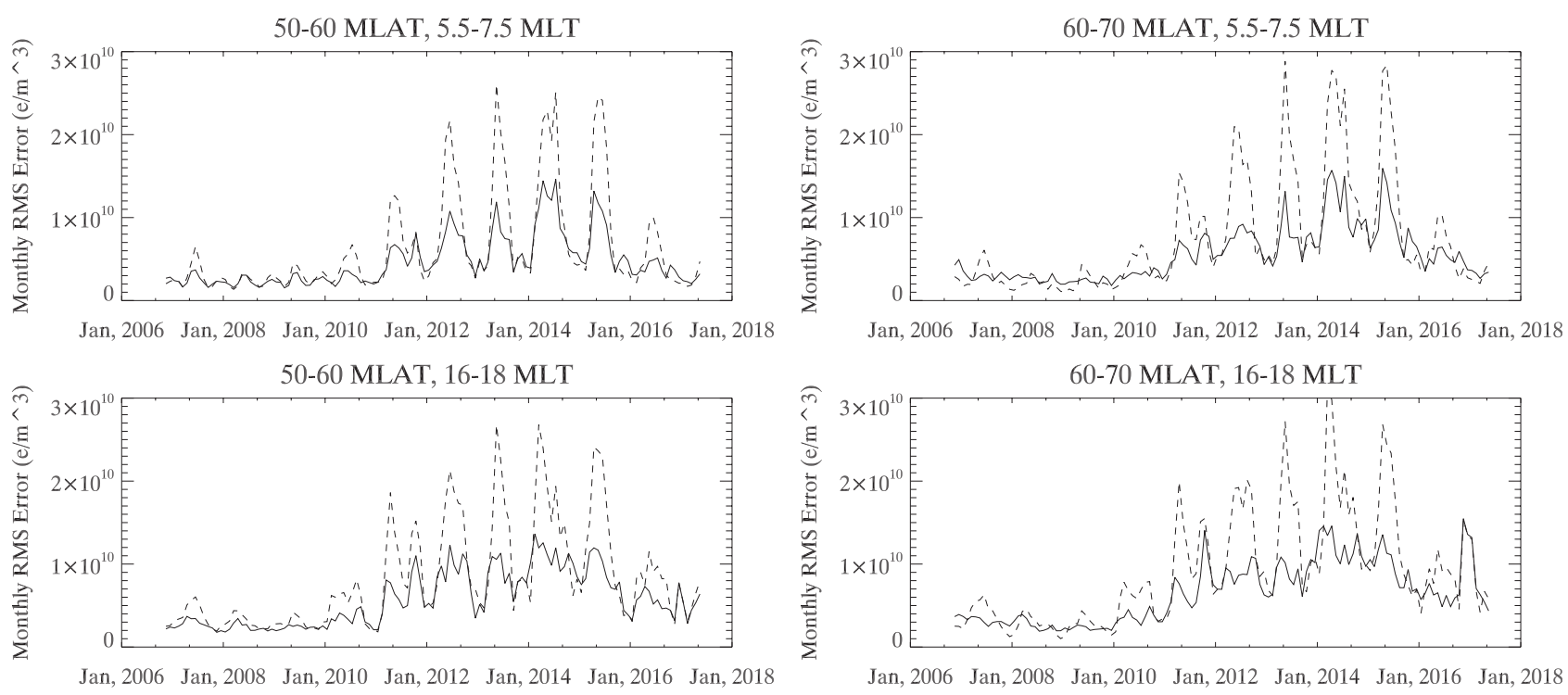

Fig. 6. Monthly RMS errors in E-CHAIM (solid lines) and IRI (dashed lines) electron density at the DMSP F17 satellite. The top plots correspond to the 5.5-7.5 MLT bin, the bottom plots correspond to the 16-18 MLT bin, the left plots are for the $50^{\circ}-60^{\circ} \mathrm{N}$ geomagnetic latitude bin, and the right plots are for the $60^{\circ}-70^{\circ} \mathrm{N}$ geomagnetic latitude bin.
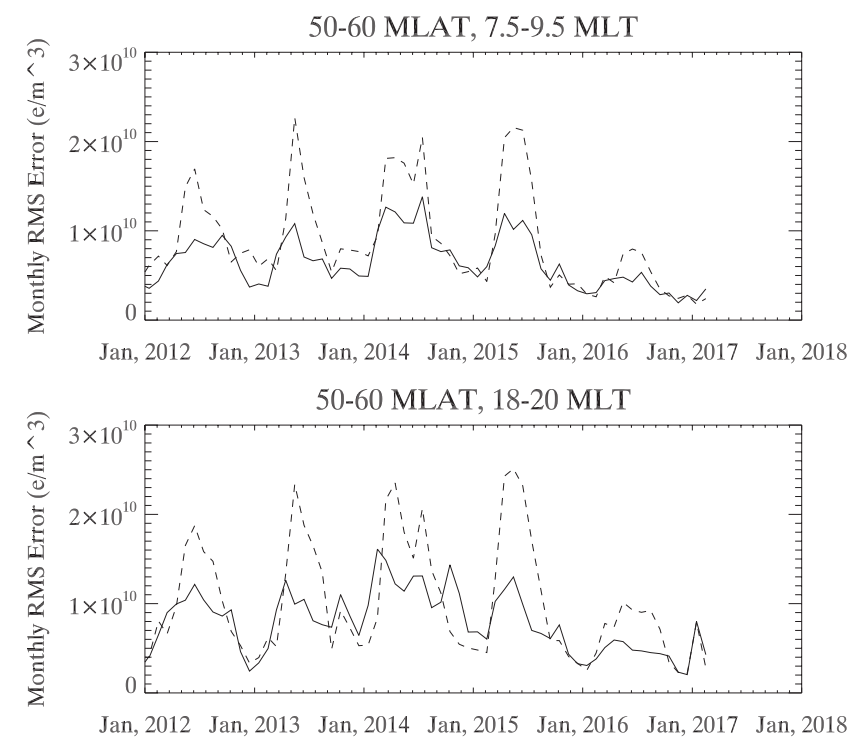

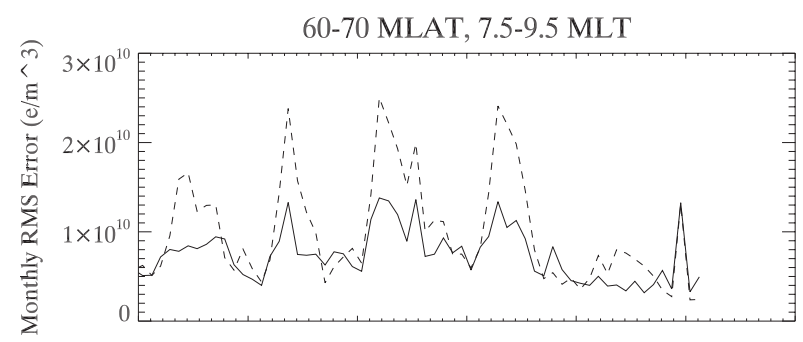

Jan, 2012 Jan, 2013 Jan, 2014 Jan, 2015 Jan, 2016 Jan, 2017 Jan, 2018

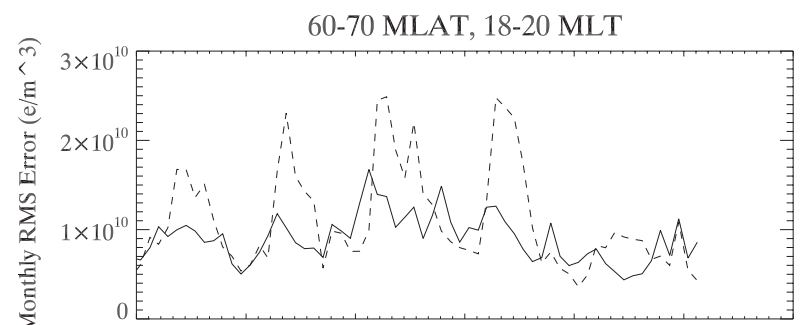

Jan, 2012 Jan, 2013 Jan, 2014 Jan, 2015 Jan, 2016 Jan, 2017 Jan, 2018

Fig. 7. Same as Figure 6 but for DMSP satellite F18. The top plots correspond to the 7.5-9.5 MLT bin, the bottom plots correspond to the 18-20 MLT bin, the left plots are for the $50^{\circ}-60^{\circ} \mathrm{N}$ geomagnetic latitude bin, and the right plots are for the $60^{\circ}-70^{\circ} \mathrm{N}$ geomagnetic latitude bin.

To better assess the performance of each model, the monthly RMS errors corresponding to the data in Figures 4 and 5 are provided in Figures 6 and 7.

Overall, the RMS errors are consistent with our observations based on Figures 4 and 5. E-CHAIM performs better than the IRI, sometimes by a factor of two or more, during summer periods. During winter periods, both models perform comparably, with the IRI performing slightly better during the winter of the declining phase of the last solar cycle. During solar minimum, both models perform comparably. In general, the errors from both models are greatest during summer periods at high solar activity and are lowest at low solar activity.

\subsection{Monthly median variability in the Polar Cap/Auroral Oval}

As the footprint of DMSP spans a wide range of MLTs at high latitudes, comparisons are approached in a slightly different manner than the other regions. In Figure 8 we present monthly median electron density from DMSP, E-CHAIM, and the IRI for all available data in a geomagnetic latitude bin of $70^{\circ}-80^{\circ} \mathrm{N}$ from satellite F17.

Corresponding plots of the measured and modeled electron density for DMSP satellite F18 are presented in Figure 9. 

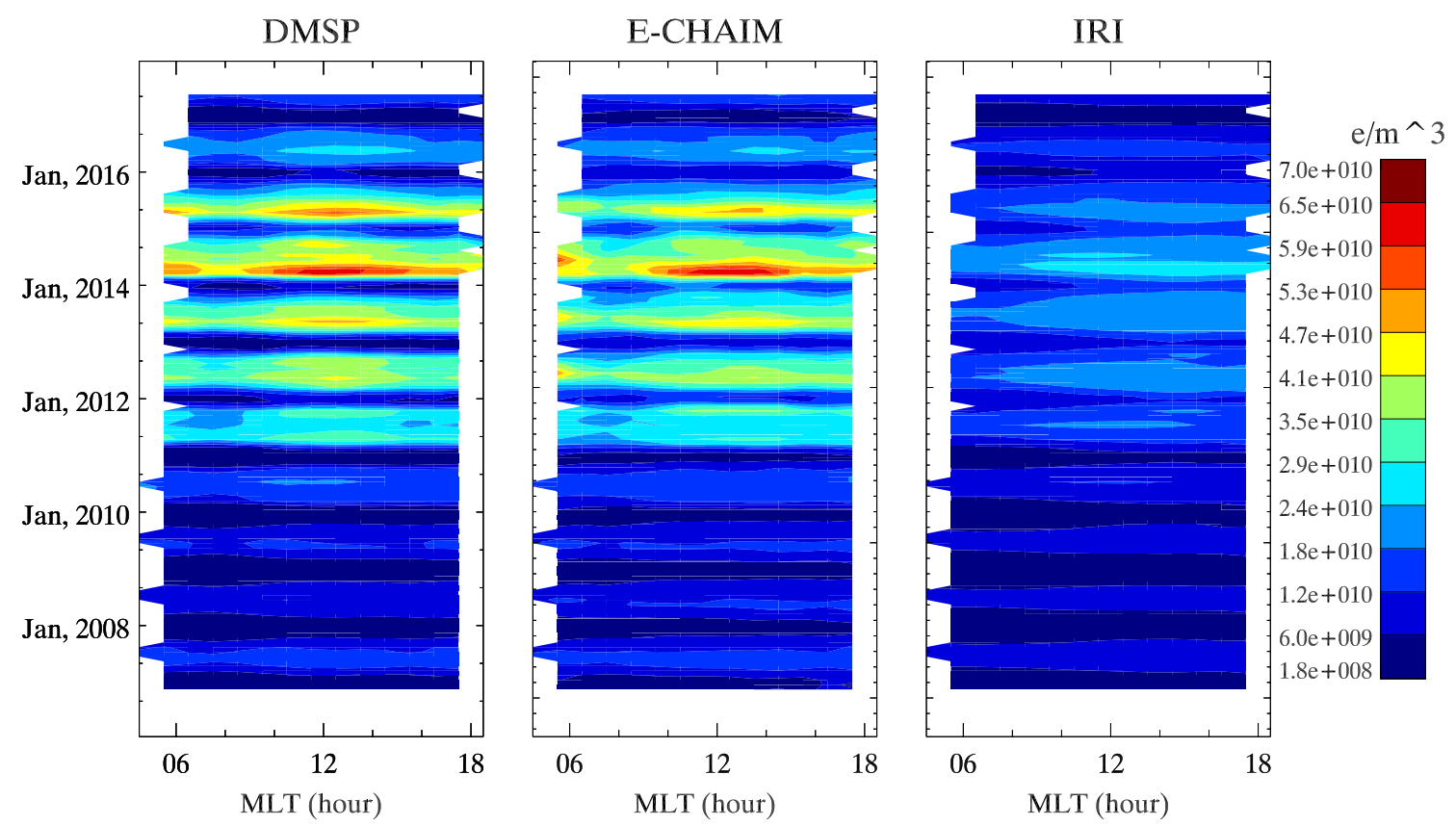

Fig. 8. Monthly median electron density from DMSP satellite F17 (left) and corresponding electron density from E-CHAIM (middle) and the IRI (right).
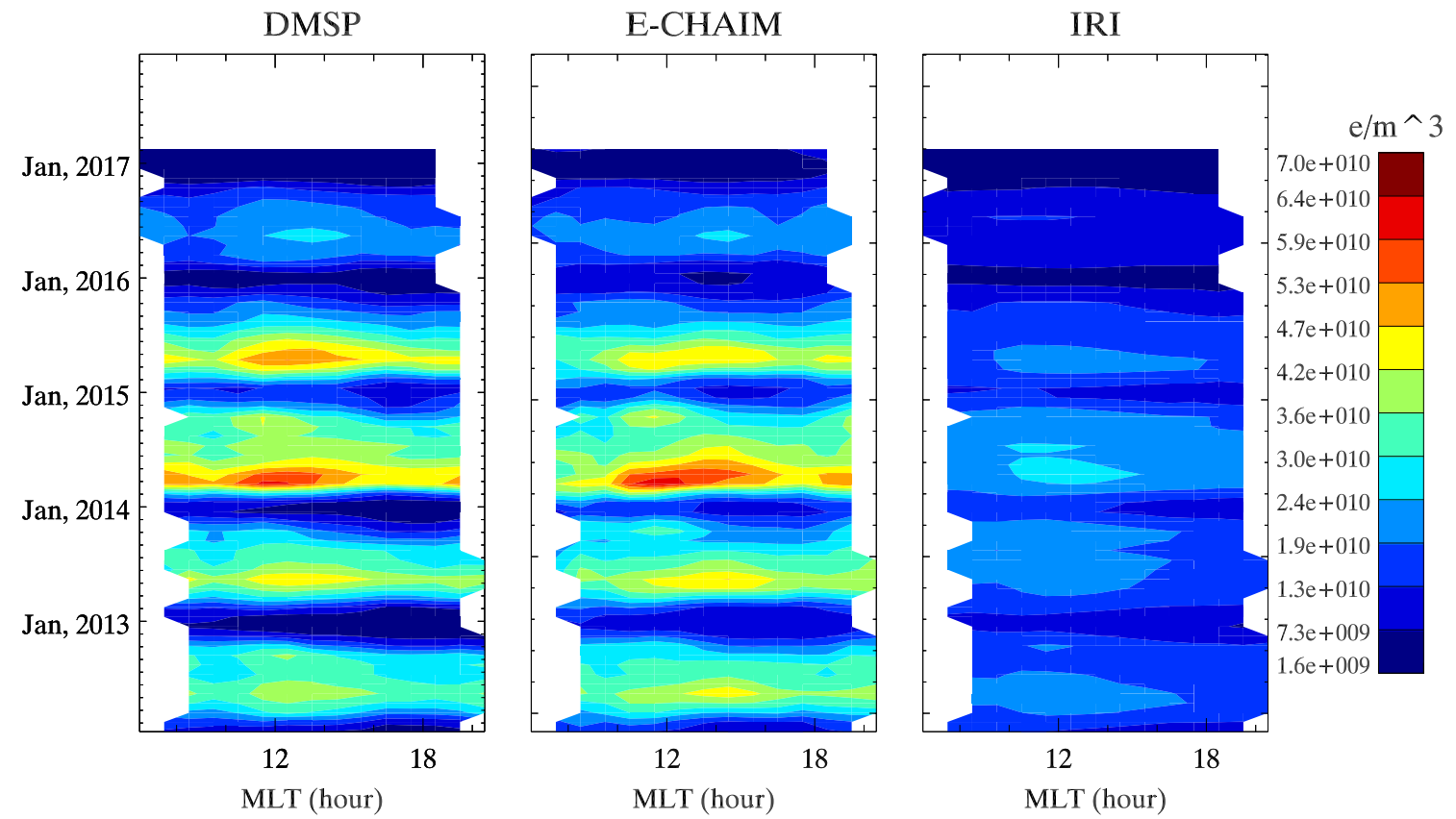

Fig. 9. Same as Figure 8 but for DMSP satellite F18.

Figures 8 and 9 show that E-CHAIM qualitatively does an excellent job at replicating DMSP F17 in situ electron density, capturing the amplitude and structure of the DMSP observation variability, but appears to overestimate electron density during winter periods. The IRI again appears to significantly underestimate summer and equinox electron density at the DMSP orbit across all MLTs but, like E-CHAIM, overestimates electron density during winter periods. To more quantitatively assess the model errors, we also present the corresponding monthly RMS errors for satellites F17 and F18 in Figures 10 and 11, respectively.

From these figures, note that both the IRI and E-CHAIM suffer their largest errors around local noon, particularly during the sudden increases in solar activity of late 2011 and early 2014. Seasonally, both models demonstrate largest errors during the equinoxes, where IRI errors exceed those of E-CHAIM by 

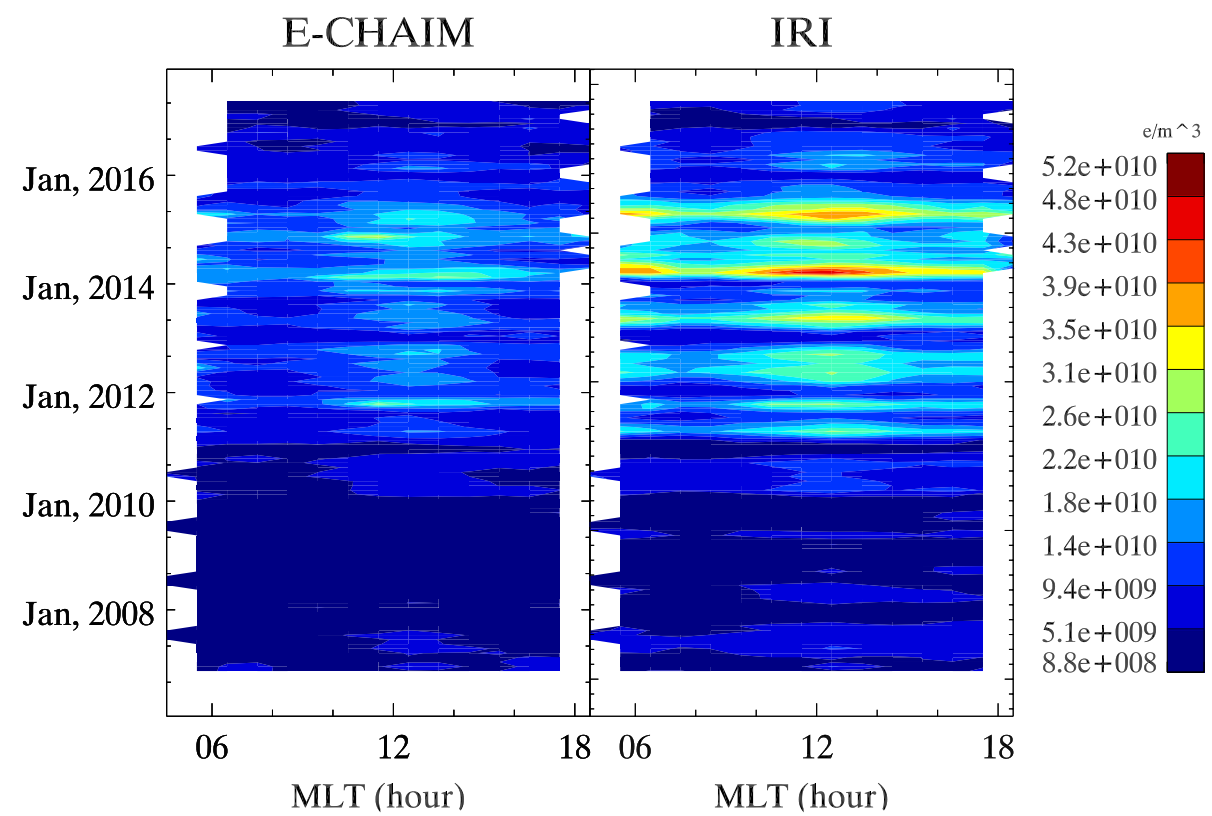

Fig. 10. Monthly RMS errors for E-CHAIM (left) and the IRI (right) for satellite F17.

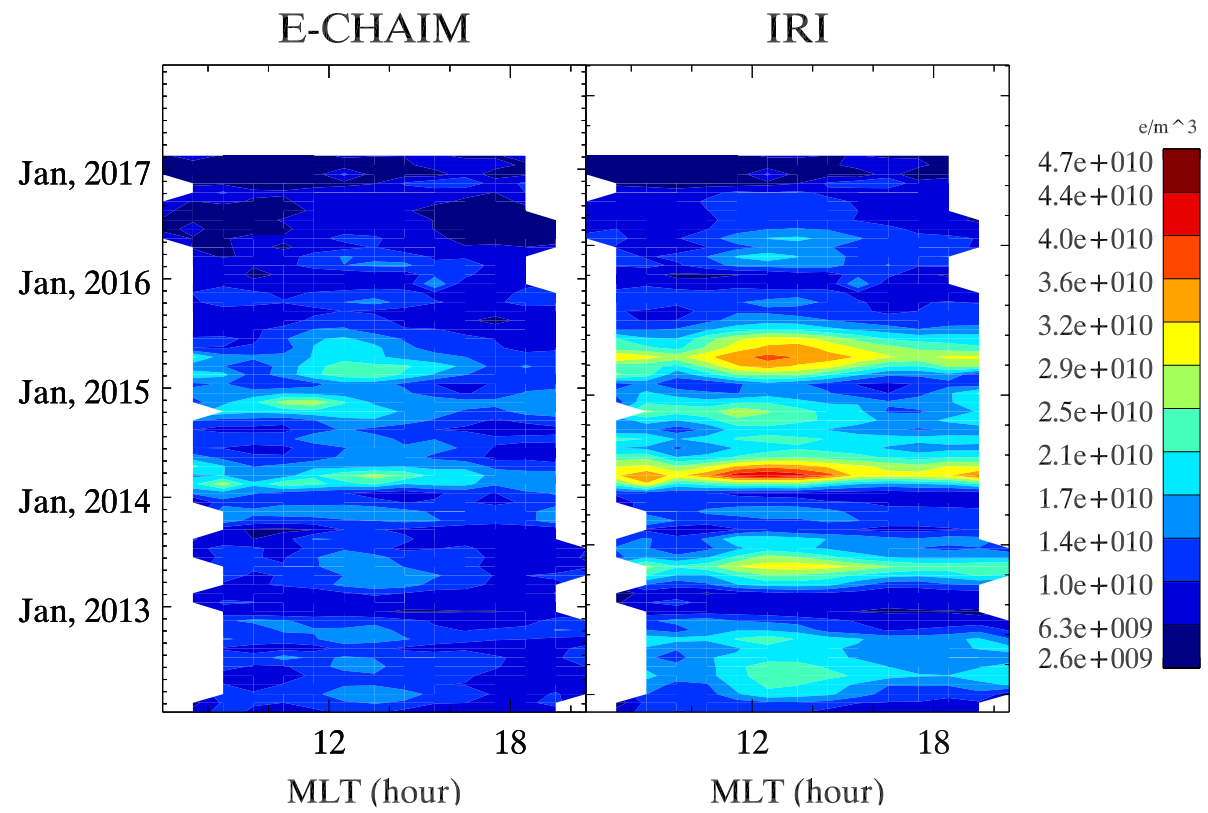

Fig. 11. Same as Figure 10 but for DMSP satellite F18.

$50-120 \%$ at high solar activity but are only slightly larger than those of E-CHAIM at low solar activity. The origin of the errors discovered in comparisons to DMSP data are diagnosed in the Discussion of Section 5.

\section{Validation of E-CHAIM using CHAMP}

To assess the performance of E-CHAIM and the IRI using CHAMP data, three geomagnetic latitude domains are considered independently: $55^{\circ}-65^{\circ} \mathrm{N}, 65^{\circ}-75^{\circ} \mathrm{N}$, and $75^{\circ}-85^{\circ} \mathrm{N}$. These bands have been chosen based on the orbital conformation of CHAMP, where the highest band was selected to center around the CHAMP polar transition. While these do not directly correspond to the bands used in the previous DMSP comparisons, we have chosen these bands to better reflect the differences in the orbits of the two satellites. In each of these bands/domains, the seasonal, solar cycle, and MLT variability of measured and modeled electron density is examined, both in terms of monthly medians and overall monthly RMS errors. 


\subsection{Overall comparison}

Prior to assessing the performance of these models within the three latitudinal domains, one must first examine their overall performance. To accomplish this, the overall distribution of errors by both models for the period between 2002 and the end of 2009 are presented in Figure 12.

Figure 12 illustrates a tendency for E-CHAIM to present a reduced bias than the IRI with a reduced median overestimation of electron density at CHAMP but a comparable or slightly wider distribution width. The overall mean difference between measured and modeled density from E-CHAIM was found to be $1.20 \times 10^{10} \mathrm{e} / \mathrm{m}^{3}$ with corresponding overall mean differences of $2.44 \times 10^{10} \mathrm{e} / \mathrm{m}^{3}$ for the IRI. In terms of overall RMS errors, E-CHAIM was found to demonstrate an RMS error of $7.11 \times 10^{10} \mathrm{e} / \mathrm{m}^{3}$ while the IRI's RMS error was found to be $7.48 \times 10^{10} \mathrm{e} / \mathrm{m}^{3}$. This relatively modest improvement in RMS errors $(\sim 5 \%)$, despite relatively substantial mean differences, suggest that E-CHAIM suffers from increased error variability, likely caused by its attempt to represent smallerscale structures, which may generate colocation errors. The IRI is highly smoothed at high latitudes and thus would not suffer such colocation errors. Purely using these overall measures, E-CHAIM appear to only modestly outperform the IRI at CHAMP orbit in high latitude regions. These comparisons are now broken down into individual geomagnetic latitude bands to further examine the spatial variability of each model's performance.

\subsection{CHAMP validation: $55^{\circ}-65^{\circ} \mathrm{N}$}

This band in geomagnetic latitude can be considered the sub-auroral zone. To begin our analysis in this band, Figure 13 presents monthly median contours of measured and modeled electron density at CHAMP.

White spaces in this, and the following, figures correspond to local times that were not sampled by the satellite due to the slow day-to-day local time shift of the satellite orbit. In Figure 13, note that both models appear to significantly overestimate electron density during nighttime periods in this latitude band at low solar activity. These errors in monthly median electron density can reach as high as an order of magnitude, exacerbated by the extremely low densities observed during these periods. Nonetheless, E-CHAIM appears to perform better than the IRI during daytime periods, better capturing the solar activity variation in daytime electron density. To examine these errors in more detail, we may consult the monthly RMS errors for both models in Figure 14.

Figure 14 clearly illustrates the qualitative differences from Figure 13. One may here note near universally better performance using E-CHAIM, particularly during daytime periods, where E-CHAIM can outperform the IRI by as much as $8 \times 10^{10} \mathrm{e} / \mathrm{m}^{3}$ near the end of 2009 .

\subsection{CHAMP validation: $65^{\circ}-75^{\circ} \mathrm{N}$}

Examining now the region largely within the auroral zone, Figure 15 presents the monthly median electron densities from CHAMP measurements and both models within this latitude band.

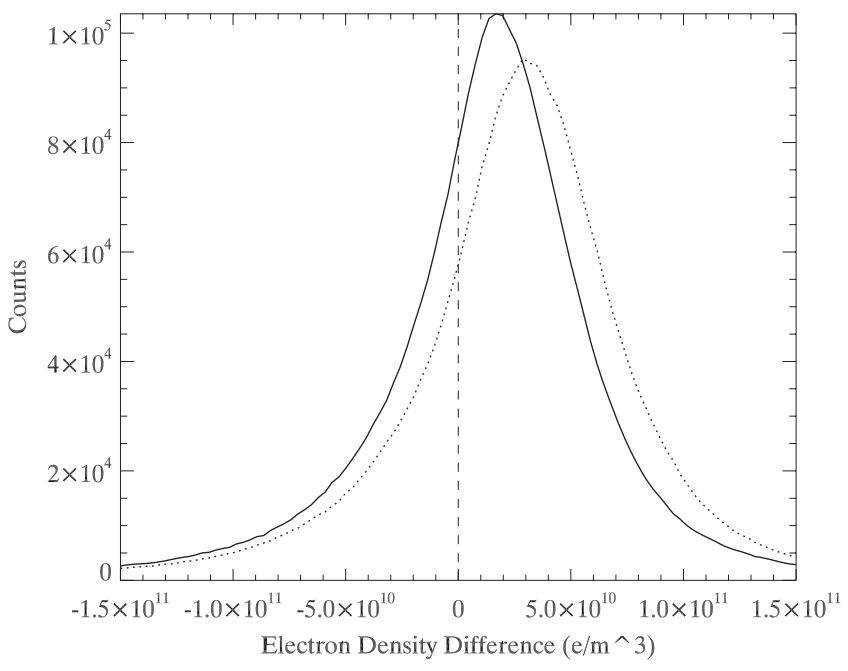

Fig. 12. Probability distributions of electron density errors for the IRI (dotted lines) and E-CHAIM (solid lines) with respect to CHAMP in situ electron density data.

From Figure 15 we note that E-CHAIM appears to overestimate nighttime electron density if the auroral oval. This is likely a manifestation of E-CHAIM overestimating the auroral enhancement in $N_{\mathrm{m}} F 2$; however, this effect will be examined in detail in future work. During these periods, the IRI appears to out-perform E-CHAIM; however, the IRI also demonstrates a tendency to overestimate day-time electron density in this region. The monthly RMS differences between the models and observations are presented in Figure 16.

In Figure 16, the improved performance of the IRI during nighttime conditions and improved performance of E-CHAIM during daytime conditions is clear, both being of similar magnitude. One may also note that E-CHAIM performs best during morning and evening periods.

\subsection{CHAMP validation: $75^{\circ}-85^{\circ} \mathrm{N}$}

For the highest latitude band (roughly within the polar cap), monthly median electron density from CHAMP and the two models is presented in Figure 17. This zone has far better local time coverage due to it encompassing the polar transit of the CHAMP satellite.

Figure 17 demonstrates a tendency for both models to overestimate electron density during winter nighttime conditions. Examining the CHAMP results we note that the diurnal maximum in electron density tends to be located in the late afternoon sector. E-CHAIM seems to capture this timing; however, the IRI appears to predict the diurnal maximum in the early morning sector, leading it to significantly overestimate electron density during these periods. Unfortunately, while E-CHAIM captures the timing of the diurnal maximum well, it tends to overestimate density at diurnal maximum and thus may suffer worse errors than the IRI during these periods. Looking at seasonal variations, both E-CHAIM and the IRI appear to underestimate the amplitude of seasonal variability in electron density at the CHAMP orbit, with the IRI barely modeling any seasonal variation during the high solar activity period between 2002 and 2005. To examine the errors in these 

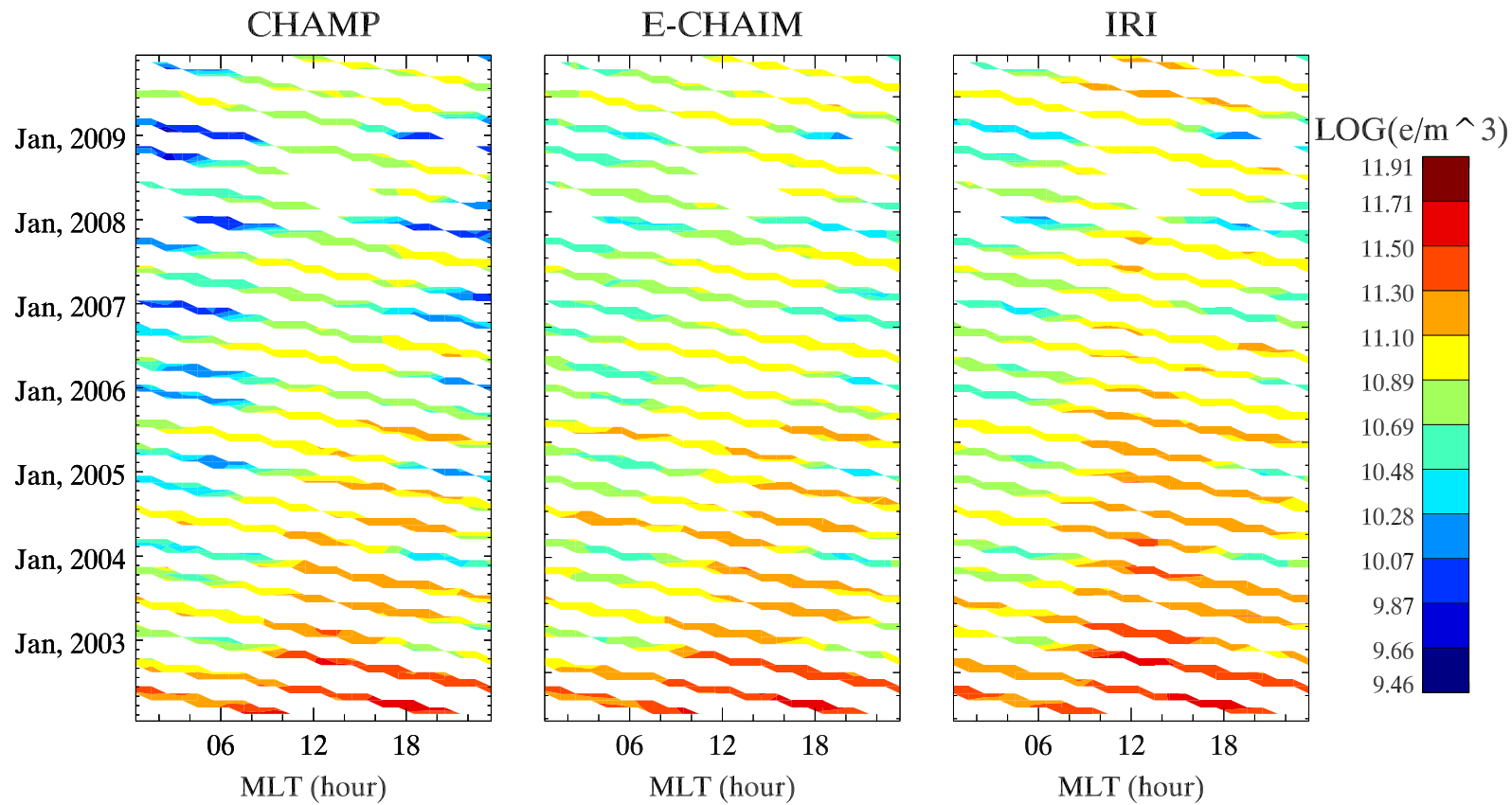

Fig. 13. Log contours of CHAMP (left), E-CHAIM (center), and IRI (right) monthly median electron density at CHAMP orbit altitude from a bin between $55^{\circ} \mathrm{N}$ and $65^{\circ} \mathrm{N}$.

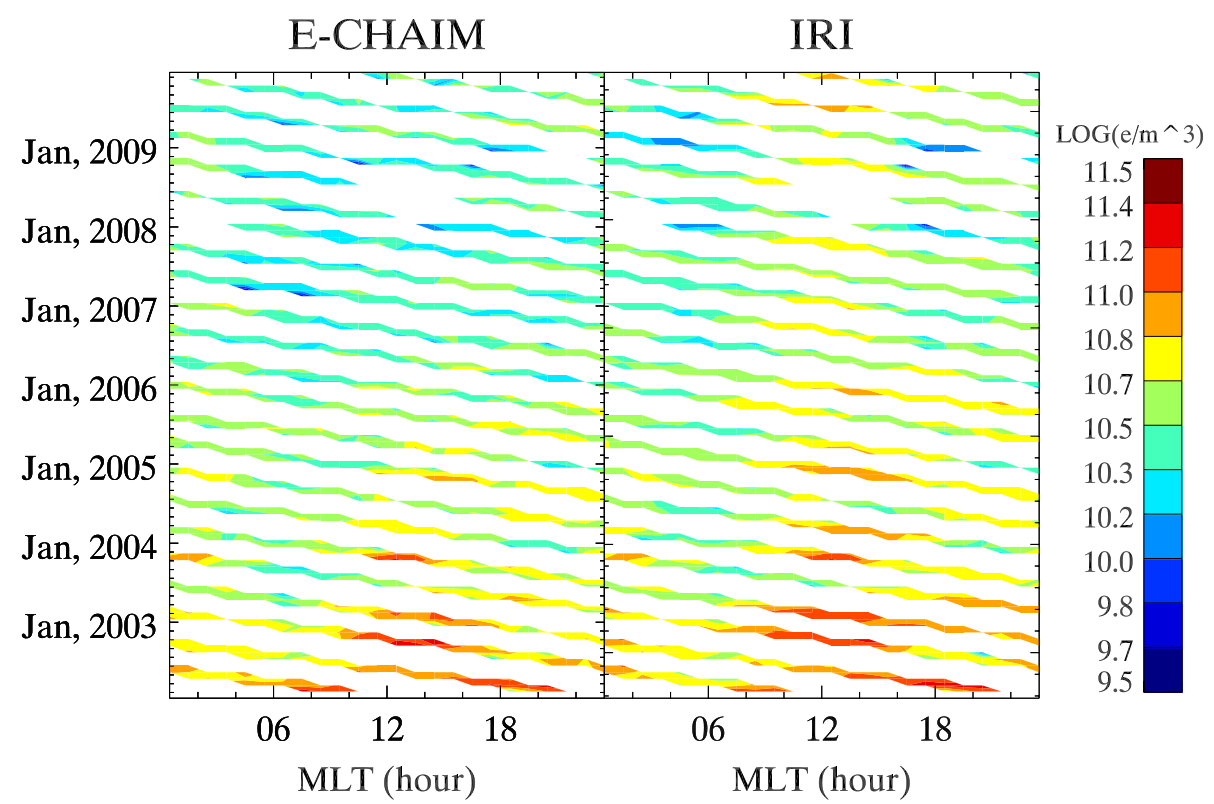

Fig. 14. Log contours of E-CHAIM (left) and IRI (right) monthly RMS electron density errors with respect to CHAMP in situ measurements from a bin between $55^{\circ} \mathrm{N}$ and $65^{\circ} \mathrm{N}$.

models more quantitatively, monthly RMS errors are presented for both models in Figure 18.

From Figure 18, it can be seen that both models mostly perform comparably at high solar activity, but E-CHAIM does demonstrate slightly worse performance by overestimating electron density during summer periods. As one tends to low solar activity, E-CHAIM appears to outperform the IRI almost universally, but particularly during nighttime conditions, where the IRI's mis-modeling of the timing of the diurnal maximum in electron density and its overestimation during winter nighttime conditions manifest.

\section{Discussion}

In general, the CHAMP-to-IRI comparisons agree with the global results of Lühr and Xiong (2010), where the IRI appears to overestimate high latitude electron density at CHAMP orbit 

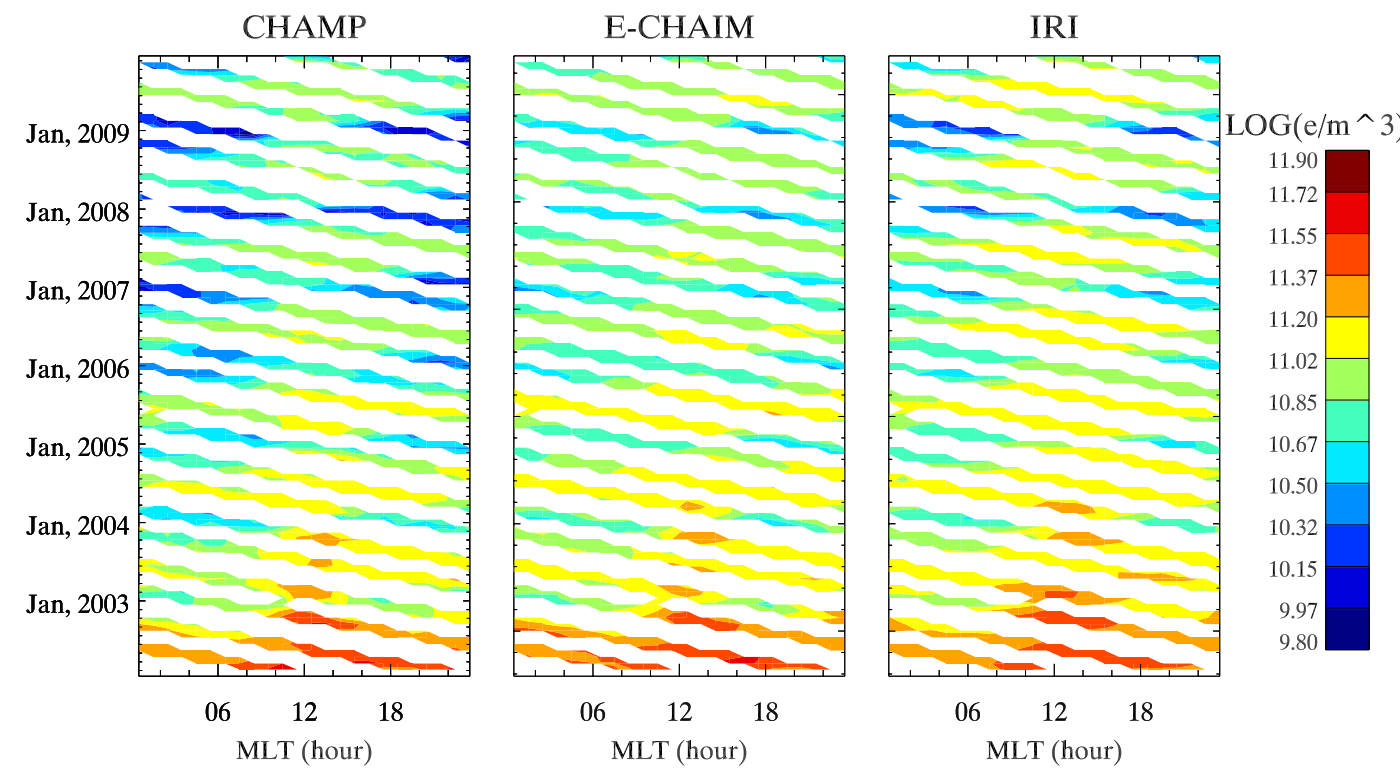

Fig. 15. Log contours of CHAMP (left), E-CHAIM (center), and IRI (right) monthly median electron density at CHAMP orbit altitude from a bin between $65^{\circ} \mathrm{N}$ and $75^{\circ} \mathrm{N}$.

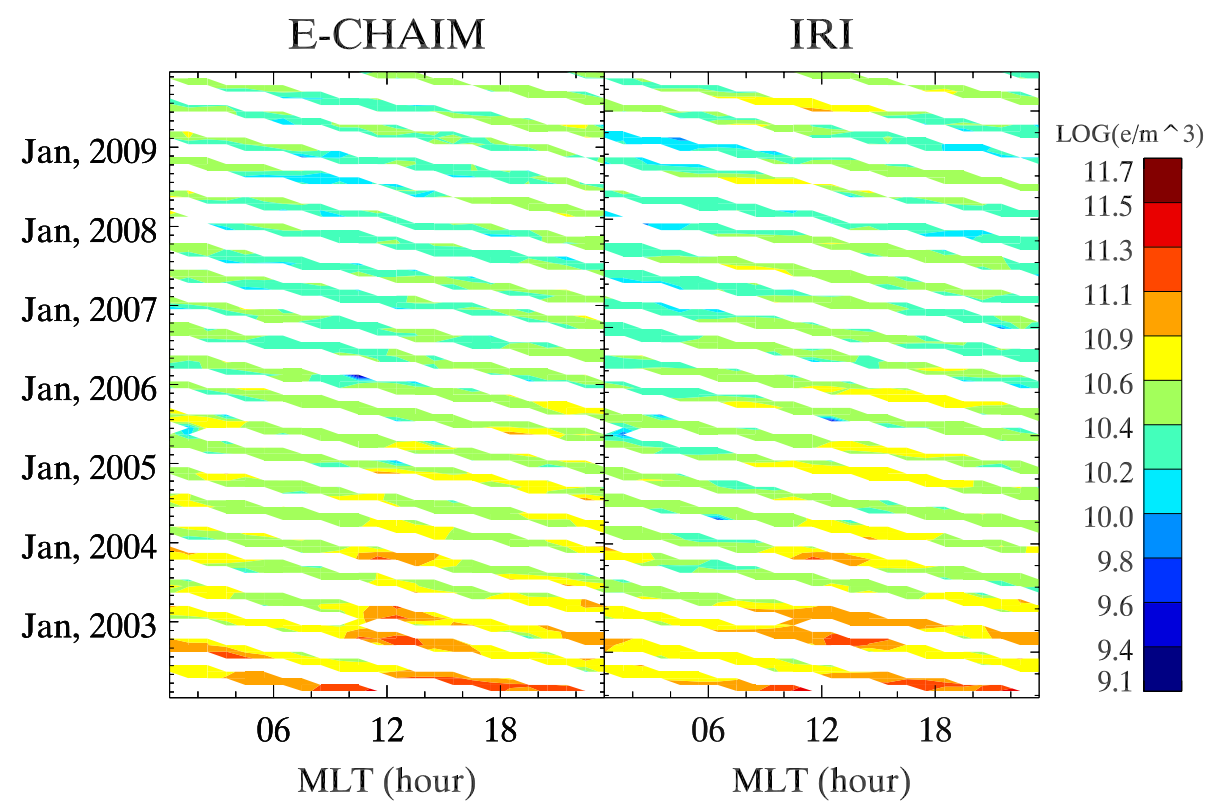

Fig. 16. Log contours of E-CHAIM (left) and IRI (right) monthly RMS electron density errors with respect to CHAMP in situ measurements from a bin between $65^{\circ} \mathrm{N}$ and $75^{\circ} \mathrm{N}$.

on average. Locally, the IRI appears to overestimate electron density almost universally, particularly during winter nighttime periods in the polar cap. The only anomaly to this overestimation is its tendency to underestimate electron density during periods of high solar activity in the polar cap equinox daytime. Overall, however, aside from relatively larger (up to $9 \times 10^{10} \mathrm{e} / \mathrm{m}^{3}$ ) overestimation errors at sub-auroral latitudes, the IRI appears to perform well at CHAMP orbit. Based on the high latitude results of Themens et al. (2014), where the IRI is demonstrated to underestimate $N_{\mathrm{m}} F 2$, and Themens et al. (2018), where it is found to overestimate the $N_{\mathrm{m}} F 2$-normalized electron density in the near peak topside, it is expected that the IRI's errors in $N_{\mathrm{m}} F 2$ and topside shape counteract each other, resulting in better overall performance at CHAMP altitudes. While the relatively larger errors in topside shape seem to have resulted in an overall overestimation trend, the IRI does perform reasonably well at these altitudes.

At CHAMP orbit, E-CHAIM is here shown to perform slightly better than the IRI at high latitudes on the overall mean, presenting a $1.2 \times 10^{10} \mathrm{e} / \mathrm{m}^{3}$ improvement over the IRI's mean error of $2.42 \times 10^{10} \mathrm{e} / \mathrm{m}^{3}$; however, E-CHAIM appears to suffer from collocation errors due to its attempt to represent smaller 

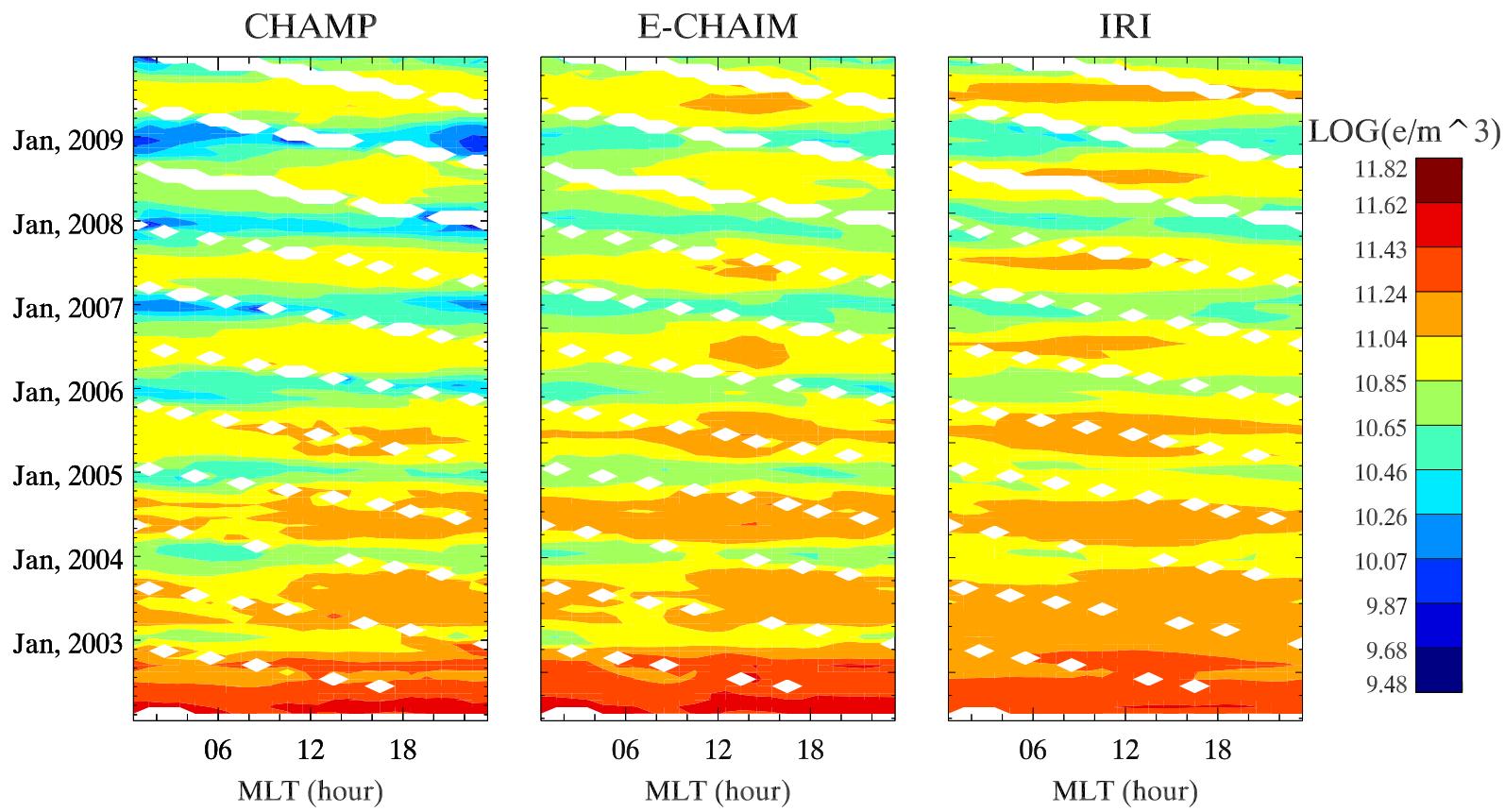

Fig. 17. Log contours of CHAMP (left), E-CHAIM (center), and IRI (right) monthly median electron density at CHAMP orbit altitude from a bin between $75^{\circ} \mathrm{N}$ and $85^{\circ} \mathrm{N}$.

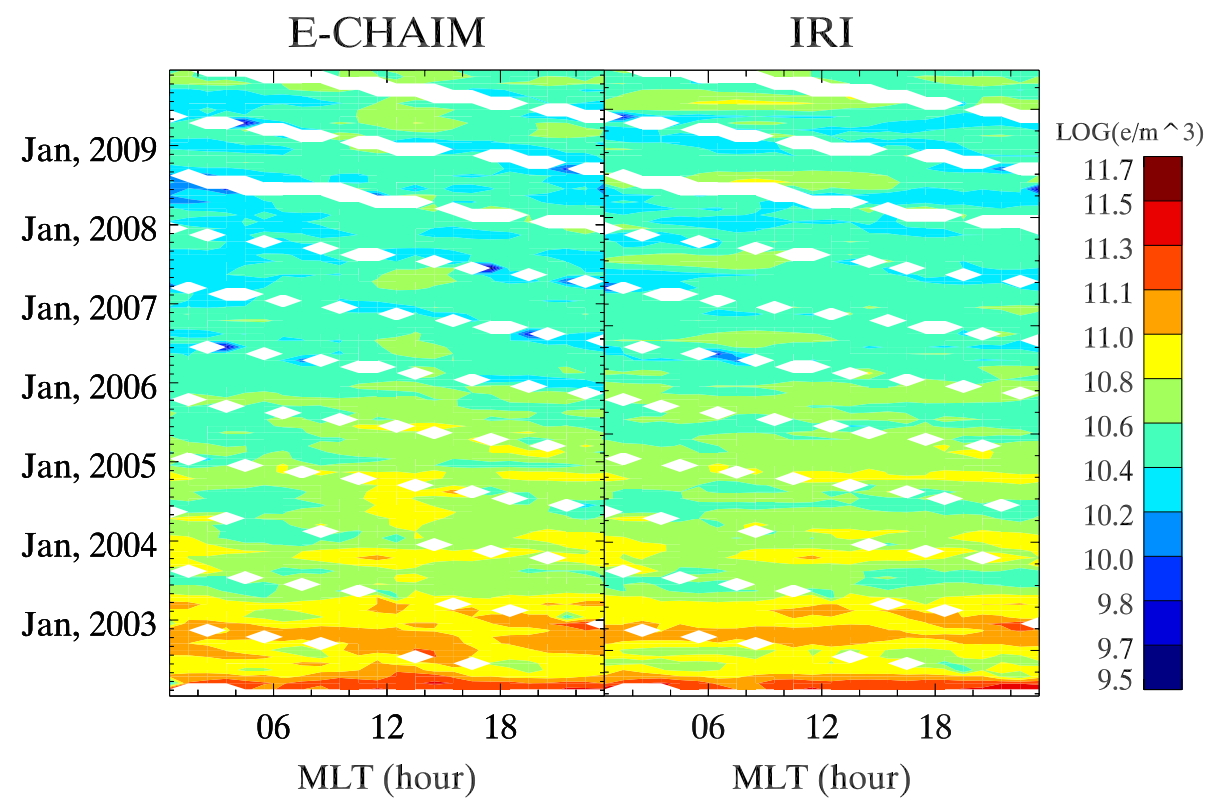

Fig. 18. Log contours of E-CHAIM (left) and IRI (right) monthly RMS electron density errors with respect to CHAMP in situ measurements from a bin between $75^{\circ} \mathrm{N}$ and $85^{\circ} \mathrm{N}$.

spatial scales than the IRI. This results in an improvement of only $3.7 \times 10^{9} \mathrm{e} / \mathrm{m}^{3}$ in terms of RMS error with respect to the IRI's RMS error of $7.48 \times 10^{10} \mathrm{e} / \mathrm{m}^{3}$. Spatially, E-CHAIM appears to outperform the IRI in the sub-auroral and polar cap regions, particularly during daytime periods. The improvement over the IRI at sub-auroral latitudes may stem from E-CHAIM's improved representation of the Main Ionospheric Trough (MIT), which is largely absent from or heavily smoothed out in the IRI (Xiong et al., 2013; Karpachev et al., 2016; Themens et al., 2017a). In the auroral zone, E-CHAIM performs comparably to, or slightly worse than, the IRI, possibly due to an overestimation of auroral $N_{\mathrm{m}} F 2$ or difficulty assigning the location of the auroral zone north-south electron density gradient. There is also a notable overestimation from both models in the representation of nighttime density in the sub-auroral zone, which may warrant investigation of whether E-CHAIM produces a 
sufficiently deep MIT. These questions will be investigated in future work.

As mentioned in Section 2.4, based on the evaluations of the IRI that demonstrated a tendency for the topside shape to overestimate electron density in the near peak and underestimate electron density at altitudes above approximately $h_{\mathrm{m}} F 2+200 \mathrm{~km}$ (Themens et al., 2018), as well as the tendency for the IRI to underestimate high latitude electron densities (Themens et al., 2014), it is expected that the IRI underestimates electron density at DMSP altitudes. This prediction appears to have been proven correct, as the IRI has been here found to nearly universally underestimate high latitude electron density at $830-880 \mathrm{~km}$ altitudes. The E-CHAIM topside model, which modified the NeQuick topside model parameterization to better represent the curvature of the high latitude topside profile, and its tendency to generally provide more accurate $N_{\mathrm{m}} F 2$ at high latitudes (Themens et al., 2017a) seems to have here culminated in strong overall performance in representing high latitude electron density in the $830-880 \mathrm{~km}$ altitude range. For both DMSP satellites tested here, E-CHAIM produced an improvement over IRI RMS errors by 3.2-3.7 × $10^{9} \mathrm{e} / \mathrm{m}^{3}(25-30 \%)$ and appears to qualitatively well capture the seasonal behaviour of DMSP in situ electron density. Based on the combined comparisons of DMSP satellites F17 and F18, the observed improvement appears to span across all magnetic local times. Nonetheless, there remain some appreciable errors in E-CHAIM electron density during winter periods at low solar activity, where performance is comparable to, but sometimes worse than, the IRI. In fact, even for periods where E-CHAIM significantly outperforms the IRI, errors can still reach as high as $20 \%$. The task, of course, remains in identifying where these remaining errors are coming from within E-CHAIM, whether it be in $h_{\mathrm{m}} F 2$, in $N_{\mathrm{m}} F 2$, or in the topside shape function. Further assessment is necessary using either independent, full electron density profile data or more in situ satellite data at varying altitudes in order to fully diagnose these errors.

The observation of the IRI significantly underestimating electron density at DMSP orbit, despite its overestimation or good performance at lower altitudes, strongly suggests that the observed errors in IRI topside electron density arise due to errors in the topside shape function and agree with previous results from Themens et al. (2018). If these errors were associated with $N_{\mathrm{m}} F 2$, topside thickness $\left(H_{0}\right)$, or $h_{\mathrm{m}} F 2$, one should see systematic and corresponding differences at both tested altitudes rather than the observed trend of overestimation near the peak of the F2-layer and underestimation in the upper topside. These results prove to mostly answer the diagnostic questions of Bilitza et al. (2012). The challenge is, however, that this is not the case in all latitude regimes. While the CHAMP results hold on average at most latitudes, the DMSP results of Migoya-Orue et al. (2013) suggest that our observed trend at DMSP orbit changes significantly at lower latitudes, with the IRI performing very well. This suggests that a correction to the NeQuick shape function that performs well at high latitudes may not be appropriate for lower latitudes, perhaps necessitating the accommodation of latitudinal variability in the NeQuick's $r$ and $g$ constants. This would also imply that the modification of the NeQuick function proposed in Themens et al. (2018) should not be used outside of the high latitude region.

\section{Conclusions}

Overall, it is here demonstrated that E-CHAIM performs well in its representation of topside electron density and, particularly at high altitudes, represents a noted improvement over the IRI. The IRI performs better near the F2-peak, where an apparent tendency to underestimate $N_{\mathrm{m}} F 2$ is countered by the tendency for the IRI to overestimate the thickness of the topside in the near-peak region. At higher altitudes, the IRI demonstrates a notable tendency to underestimate summer and equinox electron density, particularly at high solar activity where it underestimates monthly averaged electron density by as much as $60 \%$. For these same equinox and summer periods at high altitudes, E-CHAIM performs substantially better than the IRI with errors within $25 \%$. During winter periods at DMSP altitude, both models perform very comparably with RMS errors less than $1.0 \times 10^{10} \mathrm{e} / \mathrm{m}^{3}$. In terms of the regional distribution of these errors, E-CHAIM performs its best at sub-auroral latitudes, while the IRI performs its best in the auroral zone. Overall, E-CHAIM represented an improvement of $\sim 3 \times 10^{9} \mathrm{e} / \mathrm{m}^{3}$ over the IRI (see Table 1). At CHAMP altitudes, both models perform very comparably in the auroral oval and polar cap (within 5\% of each other), while E-CHAIM performs much better than the IRI in sub-auroral regions (improvements of up to $30 \%$ ). The overall performance of both models is found to be somewhat comparable at $7.11 \times 10^{10} \mathrm{e} / \mathrm{m}^{3}$ for E-CHAIM and $7.48 \times 10^{10} \mathrm{e} / \mathrm{m}^{3}$ for the IRI, where it appears that improvements in the mean representation by E-CHAIM are counteracted by a greater tendency for E-CHAIM to produce co-location errors due to its higher spatial resolution.

Acknowledgements. The authors thank H. Lühr and J. Rauberg at GFZ Potsdam for the provision and processing of the CHAMP LP data (available at: http://isdc.gfz-potsdam. de/champ/CH-ME-2-PLP). DMSP data was gathered from https://satdat.ngdc.noaa.gov/dmsp/. This study is supported, in part, under Defence Research and Development Canada contract \# W7714-186507/001/SS. Infrastructure funding for the Canadian High Arctic Ionospheric Network (CHAIN) was provided by the Canadian Foundation for Innovation and the New Brunswick Innovation Foundation. Science funding is provided by the Natural Sciences and Engineering Research Council of Canada. The editor thanks Ivan Kutiev and Sean Elvidge for their assistance in evaluating this paper. E-CHAIM source code in C, Matlab, and IDL is openly available at https://e-chaim.chain-project.net. The most recent version of E-CHAIM (v1.2.1) was used for this study.

\section{References}

Altadill D, Magdaleno S, Torta JM, Blanch E. 2013. Global empirical models of the density peak height and of the equivalent scale height for quiet conditions. Adv Space Res 52: 1756-1769. DOI: $10.1016 /$ j.asr.2012.11.018.

Bilitza D. 1990. International Reference Ionosphere 1990, NSSDC 90-22. Greenbelt, Maryland.

Bilitza D, Radicella SM, Reinisch BW, Adeniyi JO, Mosert Gonzalez ME, Zhang SR. 2000. New B0 and B1 models for IRI. Adv Space Res 25(1): 89-95. 
Bilitza D. 2001. International Reference Ionosphere 2000. Radio Sci 36(2): 261-275.

Bilitza D, Reinisch BW. 2008. International Reference Ionosphere 2007: Improvements and new parameters. Adv Space Res 42: 599-609. DOI: 10.1016/j.asr.2007.07.048.

Bilitza D, McKinnell L-A, Reinisch BW, Fuller-Rowell T. 2011. The international reference ionosphere today and in the future. $J$ Geodesy 85: 909-920. DOI: 10.1007/s00190-010-0427-x.

Bilitza D, Brown SA, Wang MY, Souza JR, Roddy PA. 2012. Measurements and IRI model predictions during the recent solar minimum. J Atmos Sol Terr Phys 86: 99-106. DOI: 10.1016/ j.jastp.2012.06.010.

Bjoland LM, Belyey V, Lovhaug UP, La Hoz C. 2016. An evaluation of International Reference Ionosphere electron density in the polar cap and cusp using EISCAT Svalbard radar measurements. Ann Geophys 34: 751-758. DOI: 10.5194/angeo34-751-2016.

Coïsson P, Radicalle SM, Leitinger R, Nava B. 2006. Topside electron density in IRI and NeQuick: Features and limitations. $A d v$ Space Res 37: 937-942.

Garner TW, Taylor BT, Gaussiran TL, Coley WR, Hairston MR, Rich FJ. 2010. Statistical behavior of the topside electron density as determined from DMSP observations: A probabilistic climatology. J Geophys Res 115: A07306. DOI: 10.1029/ 2009JA014695.

Karpachev AT, Klimenko MV, Klimenko VV, Pustovalova LV. 2016. Empirical model of the main ionospheric trough for the nighttime winter conditions. J Atmos Sol Terr Phys 146: 149-159. DOI: $10.1016 /$ j.jastp.2016.05.008.

Lühr H, Xiong C. 2010. IRI-2007 model overestimates electron density during the 23/24 solar minimum. Geophys Res Lett 37: L23101. DOI: 10.1029/2011GL045430.

Migoya-Orue YO, Radicella SM, Nava B. 2013. Comparison of topside electron density computed by ionospheric models and plasma density observed by DMSP satellites. Adv Space Res 52: 1710-1716. DOI: 10.1016/j.asr.2013.01.032.

Nava B, Coïsson P, Radicella SM. 2008. A new version of the NeQuick ionosphere electron density model. J Atmos Solar-Terr Phys 70(15): 1856-1862. DOI: 10.1016/j.jastp.2008.01.015.

Shubin VN. 2015. Global median model of the F2-layer peak height based on ionospheric radio-occultation and ground-based Digisonde observations. Adv Space Res 56: 916-928. DOI: 10.1016/j. asr.2015.05.029.

Themens DR, Jayachandran PT, Nicolls MJ, MacDougall JW. 2014. A top to bottom evaluation of IRI 2007 within the polar cap. $J$ Geophys Res Space Phys 119: 6689-6703. DOI: 10.1002/ 2014JA020052.

Themens DR, Jayachandran PT. 2016. Solar activity variability in the IRI at high latitudes: Comparisons with GPS total electron content. J Geophys Res Space Phys 121: 3793-3807. DOI: 10.1002/ 2016JA022664.

Themens DR, Jayachandran PT, Galkin I, Hall C. 2017a. The Empirical Canadian High Arctic Ionospheric Model (E-CHAIM): NmF2 and hmF2. J Geophys Res Space Phys 122: 9015-9031. DOI: $10.1002 / 2017 J A 024398$.

Themens DR, Jayachandran PT, Varney RH. 2017b. Examining the use of the NeQuick bottomside and topside parameterizations at high latitudes. Adv Space Res 68(1): 287-294. DOI: 10.1016/j. asr.2017.09.037.

Themens DR, Jayachandran PT, Bilitza D, Erickson PJ, Häggström I, Lyashenko MV, Reid B, Varney RH, Pustovalova L. 2018. Topside electron density representations for middle and high latitudes: A topside parameterization for E-CHAIM based on the NeQuick. J Geophys Res Space Phys 123(2): 1603-1617.

Xiong C, Lühr H, Ma SY. 2013. The subauroral electron density trough: Comparison between satellite observations and IRI-2007 model estimates. Adv Space Res 51: 536-544. DOI: 10.1016/j. asr.2011.09.021.

Cite this article as: Themens DR, Jayachandran P \& McCaffrey AM 2019. Validating the performance of the Empirical Canadian High Arctic Ionospheric Model (E-CHAIM) with in situ observations from DMSP and CHAMP. J. Space Weather Space Clim. 9, A21. 